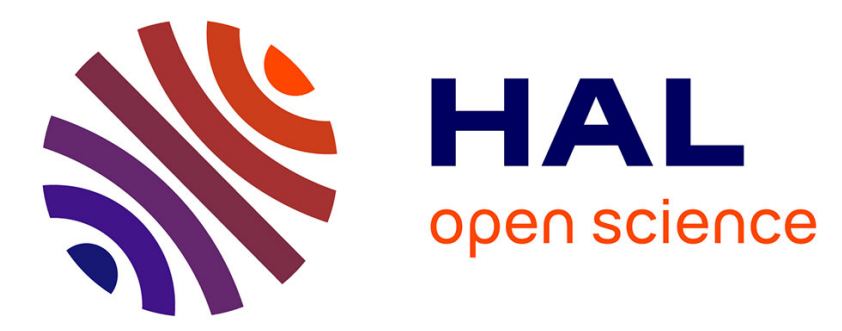

\title{
A Low-Complexity Multicarrier Scheme with LDPC Coding for Mobile-to-Mobile Environment
}

\author{
Damien Roque, Cyrille Siclet, Jean-Marc Brossier
}

\section{To cite this version:}

Damien Roque, Cyrille Siclet, Jean-Marc Brossier. A Low-Complexity Multicarrier Scheme with LDPC Coding for Mobile-to-Mobile Environment. IEEE Military Communications Conference (MILCOM12), Oct 2012, Orlando, Floride, United States. pp.n/c. hal-00723537v2

\section{HAL Id: hal-00723537 \\ https://hal.science/hal-00723537v2}

Submitted on 13 Aug 2012

HAL is a multi-disciplinary open access archive for the deposit and dissemination of scientific research documents, whether they are published or not. The documents may come from teaching and research institutions in France or abroad, or from public or private research centers.
L'archive ouverte pluridisciplinaire HAL, est destinée au dépôt et à la diffusion de documents scientifiques de niveau recherche, publiés ou non, émanant des établissements d'enseignement et de recherche français ou étrangers, des laboratoires publics ou privés. 


\title{
A Low-Complexity Multicarrier Scheme with LDPC Coding for Mobile-to-Mobile Environment
}

\author{
Damien Roque, Cyrille Siclet and Jean-Marc Brossier \\ Grenoble Images Parole Signal Automatique (GIPSA-lab) \\ 11, rue des Mathématiques \\ 38402 Grenoble, FRANCE \\ Email: \{damien.roque,cyrille.siclet,jean-marc.brossier\}@gipsa-lab.grenoble-inp.fr
}

\begin{abstract}
In this paper, we consider a mobile ad-hoc radio network in an urban area. The propagation environment between two endpoints can be modeled by a double-Rayleigh fading multipath channel. Such a mobile scenario justifies the use of filter bank based multicarrier (FBMC) transmission systems. This technique generalizes traditional cyclic prefix orthogonal frequency-division multiplexing (CP-OFDM), allowing the design of non-rectangular pulse shape filters. We show that this approach leads to a better interference mitigation in time-variant channels. We restrict our study to short filters and singletap per sub-channel equalization in order to preserve a lowcomplexity transmultiplexer. In this study, we compare FBMC with short filters to CP-OFDM in terms of coded bit-error-rate performances, using a realistic mobile-to-mobile channel model.
\end{abstract}

\section{INTRODUCTION}

The propagation constraints experienced by land tactical communication systems are usually characterized by the decentralized structure of the network and the uncertainty of the hostile environment. In practice, it results in a multipath mobile-to-mobile transmission scenario, with low rise antennas and limited a priori knowledge of the channel [1]. These assumptions reveal the need of accurate channel models that override traditional cellular models [2].

Cyclic-prefix orthogonal frequency-division multiplexing (CP-OFDM) is an attractive modulation scheme for multipath time-invariant channels since it removes inter-block interference (IBI). It relies on a low-complexity implementation using fast Fourier transform (FFT) and it allows perfect reconstruction of the transmitted signal thanks to a single-tap per subchannel equalizer [3]. However, the Doppler spread introduced by time-variant channels breaks the orthogonality between sub-channels [4, p. 753], causing inter-carrier interference (ICI). Despite this performance degradation, CP-OFDM has been adopted for several wireless mobile applications (e.g. IEEE 802.16e, 3GPP LTE Advanced).

Filter bank based multicarrier modulations (FBMC) represent a more general transmission scheme, also known as filtered multitone (FMT) or oversampled OFDM [5], [6]. This technique allows the design of various pulse shape filters, as compliant as possible with the multipath time-variant channel [7]. For example, a frequency-localized waveform may mitigate the ICI term described above. Nevertheless, this shaping is performed at the expense of a greater complexity, increasing with the filters length [8].
In this work, we focus on short prototype filters, introduced in [9]. This particular class of pulses preserves a low-complexity transmultiplexer, comparable with CP-OFDM. Furthermore, a single-tap per sub-channel equalizer may be sufficient to yield superior uncoded bit-error-rate (BER) performances in several mobile scenarios [10].

Our contributions include the development of a mobileto-mobile channel model suitable for multipath propagation in urban areas. We also discuss BER performances, through simulation results, using a low-density parity check (LDPC) code.

This paper is organized as follows. Section 2 introduces the assumptions of the mobile-to-mobile propagation in an urban environment and derives a discrete-time equivalent channel model. Section 3 presents the general transmuliplexer structure and specifies pulse shaping filters, resulting in CP-OFDM and non-rectangular orthogonal transmission schemes. Section 4 compares the BER performances of the different transmission systems, including low-density parity check (LDPC) channel coding. Finally, conclusions are presented in section 5.

\section{MoBILE-TO-MOBILE CHANNEL MODEL}

Reflections, diffraction and scattering are three physical phenomena that justify a multipath propagation beyond the line of sight (LOS) in very/ultra high frequency (V/UHF) bands. In an urban environment this non-line of sight communication (NLOS) scenario is likely to happen between two mobile transceivers.

Let $s(t)$ be the baseband equivalent transmitted signal that occupies a band $B$. We denote $\mathcal{H}$ the multipath timevarying channel with $I$ uncorrelated resolvable paths (they are mostly due to reflections in the propagation environment). The noise process $z(t)$ is a complex bandlimited Gaussian process characterized by its power spectral density $\sigma_{z}^{2} / B$ for $|f| \leq B / 2$ and 0 otherwise. The received signal is given by

$$
\begin{aligned}
r(t) & =(\mathcal{H} s)(t)+z(t) \\
& =\sum_{i=1}^{I} \alpha_{i}(t) s\left(t-\tau_{i}\right)+z(t)
\end{aligned}
$$

where $\alpha_{i}(t)$ is the time-varying complex gain of the $i$ th path with delay $\tau_{i}$. Note that we choose static delays for the 
reproducibility of simulations. They are usually standardized through experimental results (e.g. COST 207, 3GPP...).

\section{A. Double-Rayleigh model}

In a NLOS urban environment, the transmitter and the receiver are surrounded by $N_{1}$ and $N_{2}$ scatterers that can be bounded by two rings (fig. 1a). The diameter of the rings is expected to be sufficiently small to neglect the excess delay occurring in the vicinity of the transmitter and the receiver. Assuming omni-directional antennas and planar propagation, the complex coefficients associated to the $i$ th path expands as

$$
\begin{aligned}
\alpha_{i}(t)= & \sum_{n_{1}=1}^{N_{1}} \rho_{i, n_{1}}^{(1)}(t) e^{j\left(\omega_{i, n_{1}}^{(1)}(t)+\phi_{i, n_{1}}^{(1)}(t)\right)} \\
& \cdot \sum_{n_{2}=1}^{N_{2}} \rho_{i, n_{2}}^{(2)}(t) e^{j\left(\omega_{i, n_{2}}^{(2)}(t)+\phi_{i, n_{2}}^{(2)}(t)\right)}
\end{aligned}
$$

where

- $\rho_{i, n_{1}}^{(1)}(t)$ and $\rho_{i, n_{2}}^{(2)}(t)$ are the real valued gains of the $n_{1}$ th and $n_{2}$ th scatterer close to the transmitter and the receiver, respectively.

- $\omega_{i, n_{1}}^{(1)}(t)$ and $\omega_{i, n_{2}}^{(2)}(t)$ are the Doppler phase terms introduced by the motion of the transmitter and the receiver. One may develop $\omega_{n_{1}}^{(1)}(t)=2 \pi f_{1} t \cos \beta_{n_{1}}^{(1)}$ where $f_{1}$ is the maximum Doppler frequency introduced by the transmitter, $\beta_{i, n_{1}}^{(1)}$ is the angle of departure of the $n_{1}$ th ray with respect to the velocity vector $\boldsymbol{v}_{\mathbf{1}}$. A similar expansion holds for $\omega_{i, n_{2}}^{(2)}(t)$.

- $\phi_{i, n_{1}}^{(1)}(t)$ and $\phi_{i, n_{2}}^{(2)}(t)$ are phase terms depending on the carrier frequency $f_{0}$, intra-ring delays associated with each path, interactions between the waves and matter.

Without a perfect knowledge of the terrain, the signals stated above are random processes. We model $\phi_{i, n_{1}}^{(1)}(t)$ and $\phi_{i, n_{2}}^{(2)}(t)$ as independent processes, uniformly distributed over $[0,2 \pi)$. This hypothesis remains realistic in a dense scattering environment $\left(N_{1}, N_{2} \rightarrow \infty\right)$ and if the phase shifts occuring inside the rings are much larger than one [11]. Since amplitudes $\rho_{i, n_{1}}^{(1)}(t)$ and $\rho_{i, n_{2}}^{(2)}(t)$ are independent, the central limit theorem allows us to write

$$
\alpha_{i}(t)=\alpha_{i}^{(1)}(t) \alpha_{i}^{(2)}(t)
$$

where $\alpha_{i}^{(1)}(t)$ and $\alpha_{i}^{(2)}(t)$ are two independent centered Gaussian processes, characterized by their respective standard deviation $\sigma_{\alpha_{i}}^{(1)}$ and $\sigma_{\alpha_{i}}^{(2)}$. This model, referred to as doubleRayleigh fading according to the distribution of $\left|\alpha_{i}^{(1)}(t)\right|$ and $\left|\alpha_{i}^{(2)}(t)\right|$, has been justified in [12]. It can be shown from (4), that the probability density function of $\left|\alpha_{i}(t)\right|$ is

$$
f\left(\left|\alpha_{i}(t)\right|\right)=\frac{\left|\alpha_{i}(t)\right|}{\left(\sigma_{\alpha_{i}}^{(1)}\right)^{2}\left(\sigma_{\alpha_{i}}^{(2)}\right)^{2}} K_{0}\left(\frac{\left|\alpha_{i}(t)\right|}{\sigma_{\alpha_{i}}^{(1)} \sigma_{\alpha_{i}}^{(2)}}\right)
$$

where $K_{0}(\cdot)$ is the zeroth order modified Bessel function of the second kind. The phase $\arg \left\{\alpha_{i}(t)\right\}$ remains a uniform process over the interval $[0 ; 2 \pi)$, independent of the modulus [13].
A particular case has been documented earlier in [14] with the hypothesis $\rho_{i, n_{1}}^{(1)}(t)=\rho_{i, n_{2}}^{(2)}(t)=1$ and $n_{1}=n_{2}$. Such a scenario relies on the approximation of a single incoming ray per Rx scatterer, leading to single Rayleigh fading model (fig. 1b).

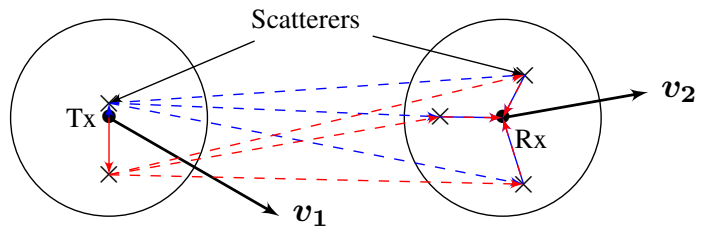

(a) Full scattering configuration.

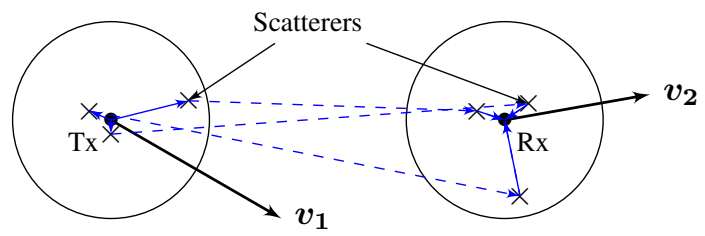

(b) Limited scattering configuration.

Fig. 1: Double scattering in a mobile-to-mobile transmission scenario (a small subset of scatterers is displayed).

\section{B. Double-Jakes power spectrum}

We first consider a displacement of the receiver over a (small) distance $\xi$ along the axis $x$ (fig. 2). Let $\phi_{i, n_{2}}^{(2)}(t)$ be the initial phase at point $x_{1}$ of the path induced by the $n_{2}$ th scatterer. Assuming a constant speed $v_{2}$ and denoting $\Delta t=\xi / v_{2}$, the receiver reaches $x_{2}$ at time $t+\Delta t$, and the phase term can be written

$$
\phi_{i, n_{2}}^{(2)}(t+\Delta t)=\phi_{i, n_{2}}^{(2)}(t)+2 \pi f_{2} \Delta t \cos \beta_{i, n_{2}}^{(2)^{\prime}} .
$$

At the transmitter side, the same displacement scenario yields a similar result in terms of phase offset with respect to the maximum Doppler frequency $f_{1}$ and the angle of departure $\beta_{i, n_{1}}^{(1)^{\prime}}$.

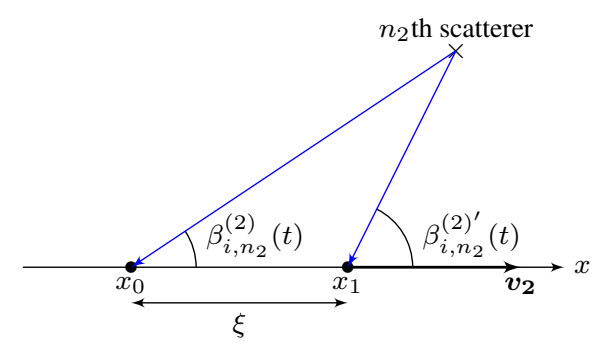

Fig. 2: Motion of the receiver over a distance $\xi$.

Wide sense stationarity is usually expected in order to establish a suitable statistical model for randomly time-variant channels. This assumption allows a time-independent formulation of $\alpha_{i}(t)$ correlation function, so that

$$
\begin{aligned}
\Gamma_{i}(\Delta t)= & \mathrm{E}\left\{\overline{\alpha_{i}^{(1)}(t)} \alpha_{i}^{(1)}(t+\Delta t)\right\} \\
& \cdot \mathrm{E}\left\{\overline{\alpha_{i}^{(2)}(t)} \alpha_{i}^{(2)}(t+\Delta t)\right\}
\end{aligned}
$$


where $\mathrm{E}\{\cdot\}$ denotes the expectation operator and $\vec{r}$ is the complex conjugate operator. Confined scattering areas and small displacement $\xi$ justify invariant amplitudes over a time $\Delta t$, namely

$$
\begin{aligned}
& \rho_{i, n_{1}}^{(1)}(t+\Delta t) \approx \rho_{i, n_{1}}^{(1)}(t) \\
& \rho_{i, n_{2}}^{(2)}(t+\Delta t) \approx \rho_{i, n_{2}}^{(2)}(t) .
\end{aligned}
$$

If we recall the phase terms independence, we obtain

$$
\begin{aligned}
\Gamma_{i}(\Delta t)= & \frac{\left(4 \sigma_{\alpha_{i}}^{(1)} \sigma_{\alpha_{i}}^{(2)}\right)^{2}}{N_{1} N_{2}} \sum_{n_{1}=1}^{N_{1}} \mathrm{E}\left\{e^{j 2 \pi f_{1} \Delta t \cos \beta_{i, n_{1}}^{(1)^{\prime}}}\right\} \\
& \cdot \sum_{n_{2}=1}^{N_{2}} \mathrm{E}\left\{e^{j 2 \pi f_{2} \Delta t \cos \beta_{i, n_{2}}^{(2)^{\prime}}}\right\} .
\end{aligned}
$$

As explained in [15], the large number of scatterers and the use of isotropic antennas allow us to write

$$
\begin{aligned}
\Gamma_{i}(\Delta t)= & \frac{\left(4 \sigma_{\alpha_{i}}^{(1)} \sigma_{\alpha_{i}}^{(2)}\right)^{2}}{4 \pi^{2}} \int_{-\pi}^{\pi} e^{j 2 \pi f_{1} \Delta t \cos \beta_{i, n_{1}}^{(1)^{\prime}}} \mathrm{d} \beta_{i, n_{1}}^{(1)^{\prime}} \\
& \cdot \int_{-\pi}^{\pi} e^{j 2 \pi f_{2} \Delta t \cos \beta_{i, n_{2}}^{(2))^{\prime}} \mathrm{d} \beta_{i, n_{2}}^{(2))^{\prime}}} \\
= & \left(4 \sigma_{\alpha_{i}}^{(1)} \sigma_{\alpha_{i}}^{(2)}\right)^{2} J_{0}\left(2 \pi f_{1} \Delta t\right) J_{0}\left(2 \pi f_{2} \Delta t\right)
\end{aligned}
$$

where $J_{0}(\cdot)$ is the zeroth order Bessel function of the first kind. The Fourier transform of $\Gamma_{i}(\Delta t)$ with respect to $\Delta t$ is referred to as the Doppler spectrum and describes the statistical distribution of motion-induced frequency shift. As explained in [14], this function is written as

$$
S(f)=\frac{\left(4 \sigma_{\alpha_{i}}^{(1)} \sigma_{\alpha_{i}}^{(2)}\right)^{2}}{\pi^{2} \sqrt{f_{1} f_{2}}} K\left(\sqrt{\frac{\left(f_{1}+f_{2}\right)^{2}-f^{2}}{4 f_{1} f_{2}}}\right)
$$

where $K(\cdot)$ is the complete elliptic integral of the first kind. The resulting power spectrum presents a "U" shape with infinite values at $|f|=f_{1}-f_{2}$ (fig. 3).

To summarize, the mobile-to-mobile non-LOS model is more stringent than the traditional base-to-mobile model in terms of fading characteristics because $\alpha_{i}(t)$ is the product of two centered Gaussian processes. As for the Doppler shift, the mobile-to-mobile scenario introduces more degrees of freedom than base-to-mobile scenario. For example, if two mobile stations move at the same speed, a null relative velocity vector may exist (i.e. no Doppler shift).

\section{Discrete-time equivalent model}

For simulation purpose, it is necessary to develop a discretetime version of the channel model denoted $\tilde{\mathcal{H}}$. Since $s(t)$ and $z(t)$ are bandlimited signals, the Whittaker-Shannon interpolation formula gives

$$
\begin{aligned}
& s(t)=\sum_{k \in \mathbf{Z}} s[k] \operatorname{sinc}(B t-k) \\
& z(t)=\sum_{k \in \mathbf{Z}} z[k] \operatorname{sinc}(B t-k)
\end{aligned}
$$

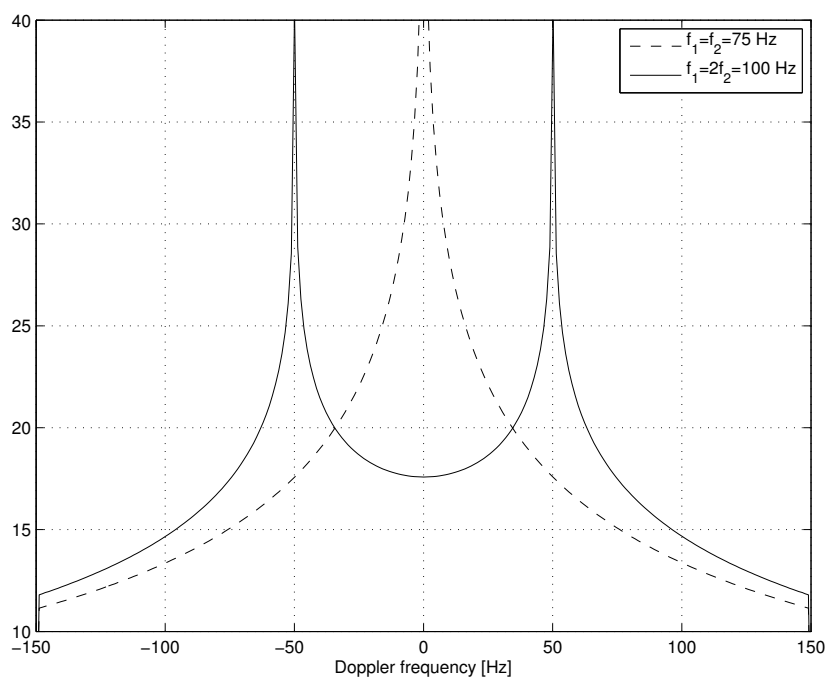

Fig. 3: Mobile-to-mobile Doppler power spectrum comparison for $f_{1} / f_{2} \in\{1,2\}$.

and the discrete-time equivalent input-output relation becomes

$$
\begin{aligned}
r[k] & =(\tilde{\mathcal{H}} s)[k]+z[k] \\
& =\sum_{l \in \mathbf{Z}} \tilde{\alpha}_{l}[k] s[k-l]+z[k]
\end{aligned}
$$

where

$$
\tilde{\alpha}_{l}[k]=\sum_{i=1}^{I} \alpha_{i}\left(\frac{k}{B}\right) \operatorname{sinc}\left(B \tau_{i}-l\right) .
$$

If we consider a number $I$ of uncorrelated $\alpha_{i}(t)$ coefficients, the overlying discrete-equivalent model provides an infinite number of $\tilde{\alpha}_{l}[k]$. Furthermore, the $1 / B$-spaced channel taps are no longer uncorrelated and we have

$$
\mathrm{E}\left\{\overline{\tilde{\alpha}_{l}[k]} \tilde{\alpha}_{l^{\prime}}\left[k^{\prime}\right]\right\}=\Omega_{l, l^{\prime}}\left[k-k^{\prime}\right]
$$

where, from (7), the correlation coefficient expands as

$\Omega_{l, l^{\prime}}\left[k-k^{\prime}\right]=\sum_{i=1}^{I} \operatorname{sinc}\left(B \tau_{i}-l\right) \operatorname{sinc}\left(B \tau_{i}-l^{\prime}\right) \Gamma_{i}\left(\frac{k-k^{\prime}}{B}\right)$.

In practice, the sequence $\left\{\tilde{\alpha}_{l}[k]\right\}_{l \in \mathbf{Z}}$ is truncated to $L$ taps whenever $\left\{\left|\tilde{\alpha}_{l}[k]\right|^{2}\right\}_{l>L} \approx 0[16]$.

\section{FBMC TRANSMISSION SYSTEM WITH SHORT FILTERS}

Faced with a time-frequency selective channel, several studies have proven the interest of FBMC transmission systems in order to mitigate ISI and ICI [5], [7], [8]. The design of the transmultiplexer is usually performed at the expense of an increased complexity brought by non-rectangular pulse shape filters (often overlapping between symbols) and the overlying matched equalizer.

As a trade-off between interference reduction and complexity, our approach focuses on short prototype filters (i.e. non-overlapping pulses) with a single-tap per sub-channel equalizer. A previous study showed attractive results in terms 


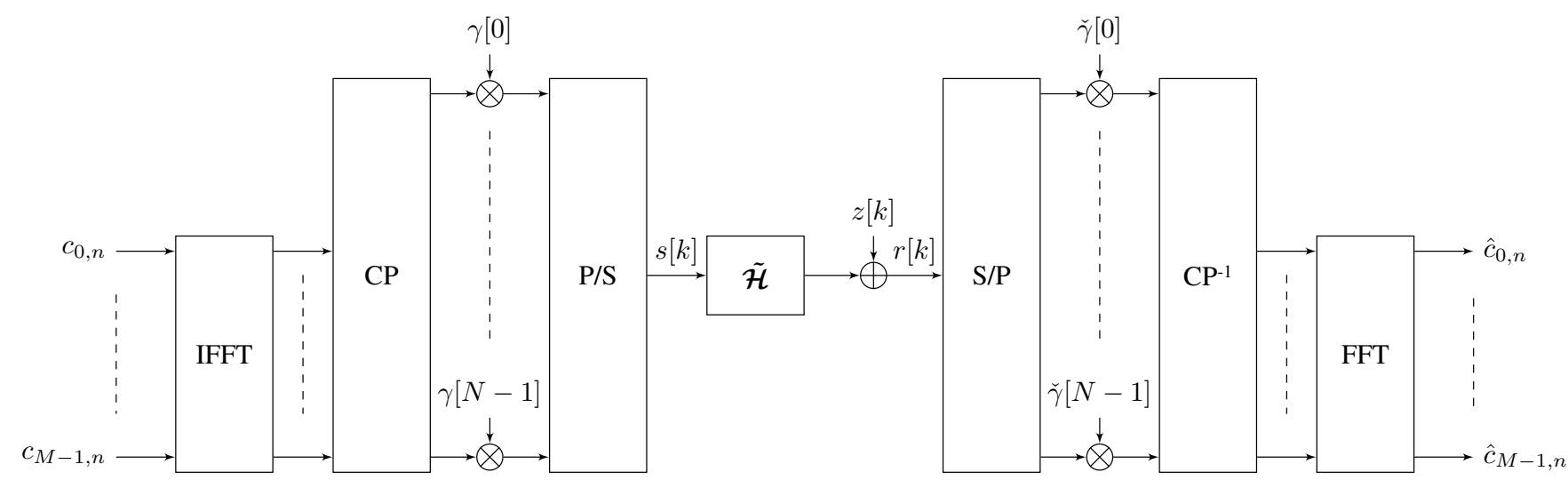

Fig. 4: Efficient implementation of a FBMC transmultiplexer with short filters.

of uncoded BER performances, in the case of base-to-mobile scenario [10].

\section{A. General transmultiplexer structure}

Information is carried by a sequence of complex symbols denoted $\left\{c_{m, n}\right\}_{(m, n) \in \boldsymbol{\Lambda}}$ where $\boldsymbol{\Lambda}=\{0, \ldots, M-1\} \times \mathbf{Z}$. The symbols are assumed to be independent and identically distributed. Their mean power is denoted $\sigma_{c}^{2}=\mathrm{E}\left\{\left|c_{m, n}\right|^{2}\right\}$.

Each $c_{m, n}$ is placed in the time-frequency plane at coordinates $(m / M, n N)$ where $M$ is the number of sub-channels and $N$ represents the number of sample per sub-channel symbol period. The oversampling ratio is given by $N / M$. The transmitted signal can be written

$$
s[k]=\sum_{(m, n) \in \mathbf{\Lambda}} c_{m, n} \gamma_{m, n}[k], \quad k \in \mathbf{Z}
$$

where $\gamma_{m, n}[k]$ is a time-frequency shifted version of the prototype pulse $\gamma[k]$ defined as

$$
\gamma_{m, n}[k]=\frac{1}{\sqrt{M}} \gamma[k-n N] e^{j 2 \pi \frac{m}{M} k}, \quad \gamma[k] \in \mathbf{R} .
$$

A necessary condition for perfect reconstruction of the transmitted symbols is the linear independence of the vectors $\left\{\gamma_{m, n}\right\}_{(m, n) \in \boldsymbol{\Lambda}}$. This requires $N / M \geq 1$, but orthogonality and completeness are not mandatory [5].

Let $x$ and $y$ be two sequences of $\ell_{2}(\mathbf{Z})$, we define the inner product $\langle x, y\rangle=\sum_{k \in \mathbf{Z}} \overline{x[k]} y[k]$, its induced norm $\|x\|=$ $\sqrt{\langle x, x\rangle}$ and the infinity norm $\|x\|_{\infty}=\max \{|x[k]|\}_{k \in \mathbf{Z}}$. At the receiver side, the dual family of $\left\{\gamma_{m, n}\right\}_{(m, n) \in \Lambda}$ is written $\left\{\check{\gamma}_{m, n}\right\}_{(m, n) \in \Lambda}$ and we have

$$
\check{\gamma}_{m, n}[k]=\frac{1}{\sqrt{M}} \check{\gamma}[k-n N] e^{j 2 \pi \frac{m}{M} k}, \quad \check{\gamma}[k] \in \mathbf{R} .
$$

The biorthogonality condition ensures perfect reconstruction of the transmitted symbols, namely $\left\langle\check{\gamma}_{p, q}, \gamma_{m, n}\right\rangle=\delta_{m, p} \delta_{n, q}$ for all $(m, n)$ and $(p, q)$ in $\boldsymbol{\Lambda} ; \delta_{m, p}$ represents the Kronecker delta. We let $\boldsymbol{\Lambda}_{\mathbf{p}, \mathbf{q}}=\boldsymbol{\Lambda} \backslash\{(p, q)\}$, then the $q$ th received symbol on the $p$ th sub-channel can be written

$$
\begin{aligned}
\hat{c}_{p, q} & =\left\langle\check{\gamma}_{p, q}, r\right\rangle \\
& =\left\langle\check{\gamma}_{p, q}, \tilde{\mathcal{H}} s\right\rangle+\left\langle\check{\gamma}_{p, q}, z\right\rangle \\
& =\underbrace{c_{p, q} A_{p, q}}_{S_{p, q}}+\underbrace{\sum_{(m, n) \in \Lambda_{\mathbf{p}, \mathbf{q}}} c_{m, n} A_{m, n, p, q}}_{I_{p, q}}+Z_{p, q}
\end{aligned}
$$

where

$$
\begin{aligned}
A_{m, n, p, q}= & \left\langle\check{\gamma}_{p, q}, \tilde{\mathcal{H}} \gamma_{m, n}\right\rangle \\
= & \frac{1}{\sqrt{M}} \sum_{k \in \mathbf{Z}} \check{\gamma}[k-q N] e^{j 2 \pi \frac{(m-p)}{M} k} \\
& \cdot \sum_{l \in \mathbf{Z}} \tilde{\alpha}_{l}[k] \gamma[k-l-n N] e^{-j 2 \pi \frac{m}{M} l} \\
A_{p, q}= & \left\langle\check{\gamma}_{p, q}, \tilde{\mathcal{H}} \gamma_{p, q}\right\rangle=A_{p, q, p, q}
\end{aligned}
$$

and

$$
Z_{p, q}=\left\langle\check{\gamma}_{p, q}, z\right\rangle=\frac{1}{\sqrt{M}} \sum_{k \in \mathbf{Z}} z[k] \check{\gamma}[k-q N] e^{-j 2 \pi \frac{p}{M} k} .
$$

The expression of the received symbol splits into three terms: $S_{p, q}$ represents the useful part of the signal, $I_{p, q}$ denotes the interference term, including inter-carrier and inter-symbol interference, $Z_{p, q}$ refers to the noise filtered by the receiver.

The coefficient $A_{m, n, p, q}$ is the complex gain introduced by the system, assuming an estimated symbol $(p, q)$ and an interfering symbol $(m, n)$.

Assuming perfect knowledge of the channel coefficients, interference mitigation is performed thanks to a low-complexity equalizer, with a single-tap per sub-channel. This type of equalizer may amplify noise and interference terms when the channel experiences severe fading.

\section{B. Filters specification : short length prototypes}

This study focuses on short length prototype filters, that is to say $\gamma[k]=\check{\gamma}[k]=0$, if $k>N-1$ and $k<0$. This particular FBMC setup allows a $N$-size block processing. It leads to an efficient implementation depicted by figure 4 and documented 
in [8]. The transmission process consists in a projection of $M$ complex symbols over an exponential basis (IFFT block). This step is followed by a cyclic extension of the data block (CP block). The resulting $N$ samples are weighted by $\gamma[k]$, $0 \leq k \leq N-1$. That is why this scheme is referred to as weighted cyclic prefix (WCP)-OFDM in the general case. At the receiver side, the dual operation is performed in order to ensure the perfect reconstruction (PR) of the transmitted symbols, that is to say $\hat{c}_{m, n}=c_{m, n}$ for any $(m, n) \in \boldsymbol{\Lambda}$ assuming $r[k]=s[k]$. In the short filters case, if we set $\Delta=$ $N-M$, PR conditions reduce to

- $\gamma[k] \check{\gamma}[k]+\gamma[k+M] \check{\gamma}[k+M]=1$ for $0 \leq k \leq \Delta-1$,

- $\gamma[k] \check{\gamma}[k]=1$ for $\Delta \leq k \leq N-1$.

First of all, let us define the rectangular filters leading to the traditional CP-OFDM scheme:

$$
\begin{aligned}
& \gamma^{\mathrm{CP}}[k]= \begin{cases}1, & \text { if } 0 \leq k \leq N-1, \\
0, & \text { otherwise },\end{cases} \\
& \check{\gamma}^{\mathrm{CP}}[k]= \begin{cases}1, & \text { if } \Delta \leq k \leq N-1, \\
0, & \text { otherwise. }\end{cases}
\end{aligned}
$$

The first $\Delta$ coefficients of $\gamma^{\mathrm{CP}}$ form the cyclic prefix in order to circumvent channel-induced inter-block interference (IBI). At the receiver side, the cyclic prefix is removed thanks to the $\Delta$ first null coefficients of $\check{\gamma}^{\mathrm{CP}}$. Since $\left\langle\gamma_{m, n}^{\mathrm{CP}}, \check{\gamma}_{p, q}^{\mathrm{CP}}\right\rangle=\delta_{m, p} \delta_{n, q}$ and $\gamma^{\mathrm{CP}} \neq \check{\gamma}^{\mathrm{CP}}$, this modulation scheme constitutes a biorthogonal system. We notice that, for $\Delta \geq 1,\left\|\gamma_{m, n}^{\mathrm{CP}}\right\|^{2}>1$ whereas $\left\|\check{\gamma}_{m, n}^{\mathrm{CP}}\right\|^{2}=1$. In presence of noise, such an unbalanced amplification scenario results in a sub-optimal transmission setup.

Apart from the CP-OFDM approach, there exists nonrectangular filters that ensure perfect reconstruction. We restrict our analysis to the linear phase orthogonal case, such that $\gamma[k]=\check{\gamma}[k]=\gamma[N-1-k]$. Thus, PR conditions become

- $\gamma^{2}[k]+\gamma^{2}[k+M]=1$ for $0 \leq k \leq(\Delta-1) / 2$,

- $\gamma^{2}[k]=1$ for $\Delta \leq k \leq(N-1) / 2$.

Short orthogonal prototype filters have been proposed in [9], assuming $N / \Delta=M / \Delta+1$ and odd values of $M / \Delta$. One of them is optimized with respect to time-frequency localization (TFL) criterion (fig. 5). A closed-form expression of this prototype is given as a good approximation of the optimized impulse response for $2 \leq M / \Delta \leq 20$ :

$$
\gamma^{\mathrm{TFL}}[k]= \begin{cases}\sin \left(\frac{(2 k+1) \pi}{4 \Delta}\right), & \text { if } 0 \leq k \leq \Delta-1, \\ 1, & \text { if } \Delta \leq k \leq M-1, \\ \sin \left(\frac{(2(\Delta-k)+1) \pi}{4 \Delta}\right), & \text { if } M \leq k \leq N-1, \\ 0, & \text { otherwise. }\end{cases}
$$

Compared to rectangular pulses used in CP-OFDM, a previous study confirms the interest of WCP-OFDM with TFL filters for mobile channels [10]. The orthogonal setup also offers an optimal approach over additive white Gaussian noise channels $[4$, p. 160].
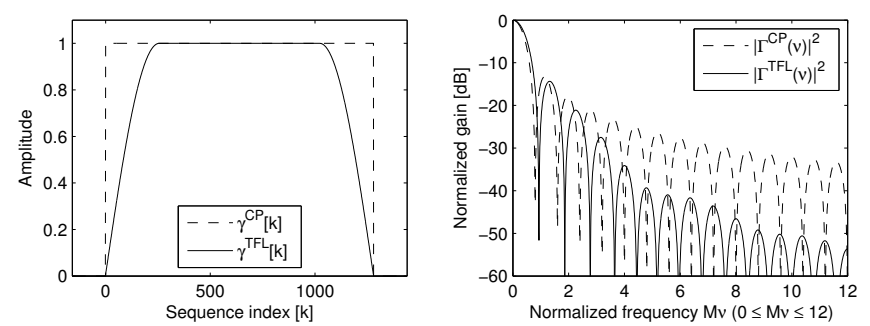

Fig. 5: Time and frequency responses of the prototype filters with $\Gamma(\nu)=1 /\|\gamma\|_{\infty} \sum_{k=0}^{N-1} \gamma[k] \exp (-j 2 \pi \nu k / N)$, using $M=1024$ and $N=1280$.

\section{BER PERFORMANCE ANALYSIS}

In this simulation framework, we consider a land mobileto-mobile transmission system, operating in an urban area. A band $B=5 \mathrm{MHz}$ is used, centered around a frequency $f_{c}=1 \mathrm{GHz}$. We use a 10-path WSSUS model where the last path occurs at $12 \mu$ s (JTCOutUrbLRLAC [17]) and whose discrete-equivalent impulse response is truncated to $L=45$ taps. This empirical model fits an outdoor propagation with low-rise buildings and low-antennas. We develop here two mobility scenarios:

- low speed, with $v_{1}=v_{2}=3 \mathrm{~km} / \mathrm{h}$;

- high speed, with $v_{1}=140 \mathrm{~km} / \mathrm{h}$ and $v_{2}=110 \mathrm{~km} / \mathrm{h}$.

We consider $M=2048$ sub-channels that carry quaternary symbols and we set $N / M \in\{17 / 16,9 / 8,5 / 4\}$. The LDPC encoder uses 32400 bit codewords and operates at a coding rate $R_{c}=3 / 4$. Each codeword is randomly interleaved and split into 8 blocks. Ideally, the transmission of each block should be spaced by a period greater than the coherence duration of the channel. In a similar way, sub-channels should by spaced by a band greater than the coherence bandwidth. Due to the time-frequency duality, it remains impossible to guarantee both conditions. In addition, applications constraints may restrict even more the channel coding scheme (e.g. interactivity, medium access control...). In this work, we choose the best diversity scenario where blocks are sent over independent channel realizations.

At the receiver side, a single-tap per sub-channel equalizer is used. After the deinterleaver, a LDPC decoder performs 10 iterations over each codeword without parity check $^{1}$. We compare the performances of CP-OFDM with TFL-OFDM (i.e. WCP-OFDM with TFL pulses). Bit-error-rate (BER) is plotted as a function of $E_{b} / N_{0}$, where $E_{b}=N \sigma_{c}^{2} / 2 M B$ and $N_{0}=\sigma_{z}^{2} / B$ (fig. 6).

First of all, we notice that specified oversampling ratios lead to $\Delta>L$. In the particular case of CP-OFDM, this condition is sufficient to cancel channel-induced IBI. However, for a general WCP-OFDM transmultiplexer, IBI may remain present.

In the low mobility scenario, we show that TFL-OFDM obtains better performances results than CP-OFDM regardless

\footnotetext{
${ }^{1}$ Such a channel coding configuration $\left(R_{c}=3 / 4,10\right.$ iterations) is chosen in order to achieve a statistically representative simulation with a reasonable computing time.
} 
of the oversampling ratio. This emphasizes the need of orthogonal signaling in mainly noisy channels. Indeed, since the TFL impulse response shows smooth transitions, it justifies a low IBI term in presence of multipath propagation. We also see that CP-OFDM performances decrease as $N / M$ increases. It summarizes the fact that the energy used to form the cyclic prefix is not recovered by the receiver.

In the high mobility scenario, the same behavior is observed for CP-OFDM. However, for high $E_{b} / N_{0}$ values, the performances of TFL-OFDM increase with $N / M$. One may show that the frequency localization of the TFL pulse increases with $N / M$. As a consequence, such a good frequency containment leads to ICI mitigation and provides a lower BER.

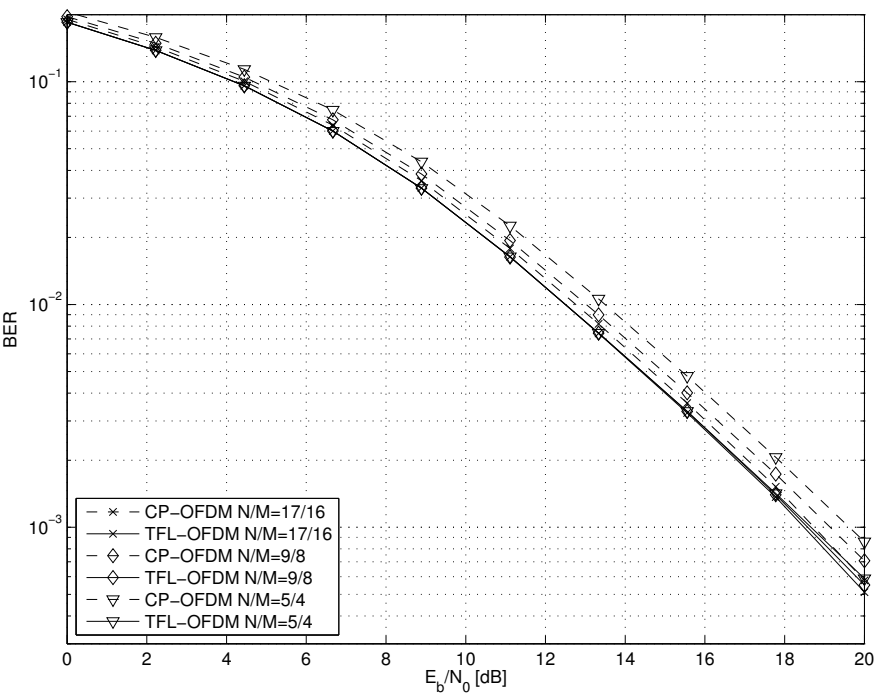

(a) BER performance in low mobility scenario.

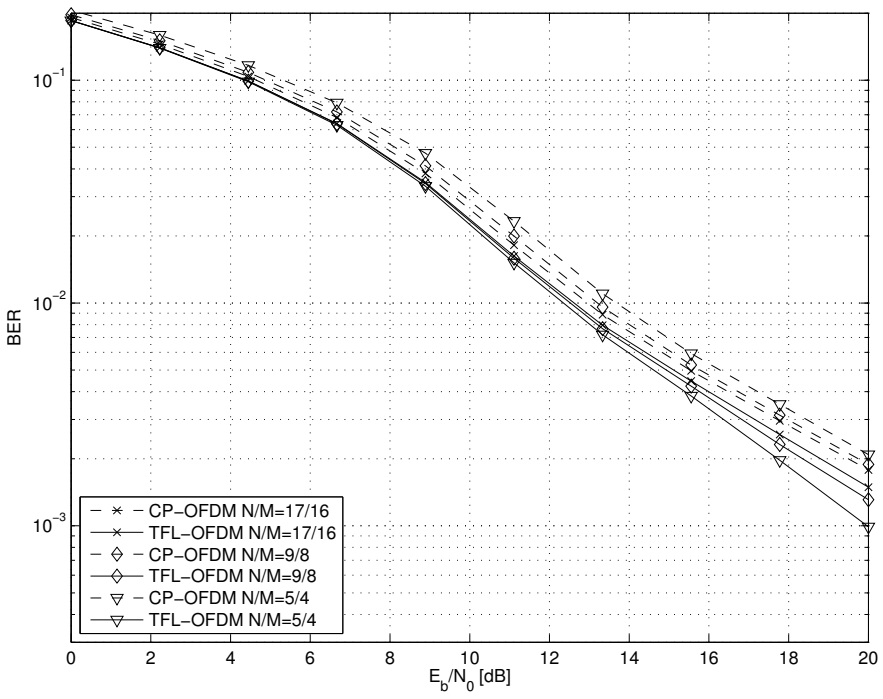

(b) BER performance in high mobility scenario.

Fig. 6: BER performances for two mobility scenarios in a mobile-to-mobile channel. Comparison between CP-OFDM and TFL-OFDM for $M=2048$ and several values of $N / M$.

\section{CONClusion}

We have defined a multipath mobile-to-mobile channel model that fits a non-LOS propagation in UHF band. We then proposed a low-complexity multicarrier scheme using short length pulse shape filters and a single-tap per sub-channel equalizer. We have shown, through simulation results, that an orthogonal transmission setup, provided with optimized TFL filters, outperforms CP-OFDM in a realistic transmission scenario. These results extend the work presented in [10], introducing a double-Rayleigh fading model and a LDPC channel coding scheme.

\section{ACKNOWLEDGMENT}

This work has been supported by the French defense procurement agency (DGA) and Orange-Labs. The authors would like to thank D. Pinchon and P. Siohan who designed the filters discussed in this article.

\section{REFERENCES}

[1] K. Bullington, "Radio propagation for vehicular communications," IEEE Trans. Veh. Technol., vol. 26, no. 4, pp. 295-308, 1977.

[2] A. Molisch, F. Tufvesson, J. Karedal, and C. Mecklenbrauker, "A survey on vehicle-to-vehicle propagation channels," IEEE Wireless Commun. Mag., vol. 16, no. 6, pp. 12-22, 2009.

[3] Z. Wang and G. B. Giannakis, "Wireless multicarrier communications," IEEE Signal Process. Mag., vol. 17, no. 3, pp. 29-48, 2000.

[4] J. Proakis and M. Salehi, Digital communications, 5th ed. McGrawHill, 2006.

[5] W. Kozek and A. F. Molisch, "Nonorthogonal pulseshapes for multicarrier communications in doubly dispersive channels," IEEE J. Sel. Areas Commun., vol. 16, no. 8, pp. 1579-1589, 1998.

[6] G. Cherubini, E. Eleftheriou, and S. Olcer, "Filtered multitone modulation for very high-speed digital subscriber lines," IEEE J. Sel. Areas Commun., vol. 20, no. 5, pp. 1016-1028, 2002.

[7] T. Strohmer and S. Beaver, "Optimal OFDM design for time-frequency dispersive channels," IEEE Trans. Commun., vol. 51, no. 7, pp. 11111122, 2003.

[8] N. Moret and A. M. Tonello, "Design of orthogonal filtered multitone modulation systems and comparison among efficient realizations," EURASIP J. Adv. Signal Process, vol. 2010, pp. 10:1-10:11, January 2010.

[9] D. Pinchon and P. Siohan, "Closed-form expressions of optimal short PR FMT prototype filters," in Proc. IEEE Global Telecommunications Conf. GLOBECOM '11, 2011.

[10] D. Roque, C. Siclet, and P. Siohan, "A performance comparison of FBMC modulation schemes with short perfect reconstruction filters," in Proc. IEEE International Conference on Telecommunications, ICT '12, 2012.

[11] R. Clarke, "A statistical theory of mobile-radio reception," Bell Syst. Tech. J, vol. 47, no. 6, pp. 957-1000, 1968.

[12] I. Z. Kovacs, P. C. F. Eggers, K. Olesen, and L. G. Petersen, "Investigations of outdoor-to-indoor mobile-to-mobile radio communication channels," in Proc. VTC 2002-Fall Vehicular Technology Conf. 2002 IEEE 56th, vol. 1, 2002, pp. 430-434.

[13] C. S. Patel, G. L. Stuber, and T. G. Pratt, "Simulation of rayleigh-faded mobile-to-mobile communication channels," IEEE Trans. Commun., vol. 53, no. 11, pp. 1876-1884, 2005.

[14] A. S. Akki and F. Haber, "A statistical model of mobile-to-mobile land communication channel," IEEE Trans. Veh. Technol., vol. 35, no. 1, pp. 2-7, 1986.

[15] C. Patel, G. Stuber, and T. Pratt, "Statistical properties of amplify and forward relay fading channels," Vehicular Technology, IEEE Transactions on, vol. 55, no. 1, pp. $1-9$, jan. 2006.

[16] C. Iskander, "A MATLAB-based object-oriented approach to multipath fading channel simulation," Hi-Tek Multisystems, Tech. Rep., 2008.

[17] K. Pahlavan and A. Levesque, Wireless information networks. Wiley Online Library, 1995. 